\title{
ESTIMATIVA DA EVAPOTRANSPIRAÇÃO DE REFERÊNCIA PELA EQUAÇÃO DE HARGREAVES-SAMANI NO ESTADO DO CEARÁ, BRASIL
} J. A. da Silva Filho ${ }^{1}$

${ }^{1}$ UFRB - Univ Federal do Recôncavo da Bahia, NEAS - Núcleo de Engenharia de Água e Solo, Campus Cruz das Almas, BA, Brasil

${ }^{2}$ UFC - Univ Federal do Ceará, DENA -Departamento de Engenharia Agrícola, Fortaleza, CE, Brasil

Article history: Received 23 April 2015; Received in revised form 03 June 2015; Accepted 08 June 2015; Available online 30 June 2015.

\section{RESUMO}

Estudos que levam em consideração os aspectos meteorológicos de uma região, sobretudo, na estimativa adequada da evapotranspiração de referência $\left(\mathrm{ET}_{\mathrm{o}}\right)$ podem contribuir para a otimização dos recursos hídricos. Diante do exposto, objetivou-se com o presente trabalho avaliar a estimativa da $\mathrm{ET}_{\mathrm{o}}$ pelo método de HARGREAVES \& SAMANI (1985) (HS) em comparação ao método padrão Penman-Monteith FAO-56 (PM-FAO 56), nas condições climáticas do estado do Ceará. A comparação dos valores de $\mathrm{ET}_{\mathrm{o}}$ obtidos pelo método padrão PM-FAO 56 e por HS foi realizada usando o erro padrão de estimativa (EPE), a razão entre as médias $(\zeta)$ e os índices de concordância "d" e desempenho "c". Quanto ao índice "c", os maiores valores foram encontrados nas localidades de Iguatu ("c" = 0,74 ; "Bom"), Jaguaruana e Crateús ("c" = 0,73; "Bom") e essa estimativa poderá melhorar com a calibração dos parâmetros da equação de HS.

Palavras-chave: Manejo da irrigação, elementos climáticos, Penman-Monteith.

\section{REFERENCE EVAPOTRANSPIRATION ESTIMATED BY HARGREAVES- SAMANI IN THE STATE OF CEARÁ, BRAZIL}

\begin{abstract}
Studies that consider the meteorological aspects of a region, especially, in adequate estimation of the reference evapotranspiration $\left(\mathrm{ET}_{\mathrm{o}}\right)$ can contribute to the optimization of water resources. In this context, in order to evaluate the estimate $\mathrm{ET}_{\mathrm{o}}$ by HARGREAVES \& SAMANI (1985) method compared to the FAO-Penman-Monteith standard method, in climatic conditions of the state of Ceará. The comparison of the values of $\mathrm{ET}_{\mathrm{o}}$ obtained by FAO-Penman-Monteith standard method and by HARGREAVES \& SAMANI (1985) was performed using the standard error of estimate (SEE), the ratio of the averages $(\zeta)$ and the index of agreement "d" and performance "c". For the index "c", the highest values were in the localities of Iguatu ("c" = 0.74, "Good"), Jaguaruana and Crateús ("c" = 0.73, "Good") and this estimate may improve with the calibration of HARGREAVES \& SAMANI (1985) equation parameters.
\end{abstract}

Keywords: Irrigation management, climatic elements, Penman-Monteith.

mairtong@ hotmail.com 


\section{INTRODUÇÃO}

O conhecimento da distribuição espacial e temporal da disponibilidade hídrica de uma região permite estabelecer diretrizes para implementação de políticas de planejamento para o uso racional da água. Diante da crescente demanda dos recursos hídricos e os conflitos entre os múltiplos usos, seja na área agrícola que demanda um volume bem maior do que nas atividades urbanas, exige que o planejamento e a gestão da sua utilização ocorram em termos racionais e otimizados.

Neste sentido, o correto conhecimento da evapotranspiração das culturas (ETc), seja para projeto e/ou manejo de irrigação, assume fundamental importância no volume de água a ser aplicado na irrigação. Uma das alternativas para se racionalizar o uso da água em projetos agrícolas, é estimar a ETc a partir da evapotranspiração de referência $\left(E_{0}\right)$ e do coeficiente de cultura $(\mathrm{Kc})$ (ESTEVES et al., 2010; MENDONÇA \& DANTAS, 2010; CARVALHO et al., 2011; TRAJKOVIĆ et al., 2011; MINUZZI et al., 2014).

$\mathrm{Na}$ escolha de um método para estimativa da $\mathrm{ET}_{\mathrm{o}}$ devem ser consideradas a praticidade e a precisão visto que, apesar dos métodos teóricos e micrometeorológicos serem baseados em princípios físicos, eles também apresentam limitações, sobretudo quanto à precisão instrumental, o que pode restringir a sua utilização. Diversos pesquisadores propuseram modelos para a estimativa da $\mathrm{ET}_{\mathrm{o}}$, com as diferentes concepções e número de variáveis envolvidas (SILVA et al., 2015).

Dentre os vários métodos existentes na literatura para estimativa da $\mathrm{ET}_{0}$, a Organização das Nações Unidas para Agricultura e Alimentação (FAO) recomendada como padrão, a equação de Penman-Monteith, publicada em seu boletim número 56 para a estimativa da $\mathrm{ET}_{\mathrm{o}}$ e calibração de outros métodos empíricos (ALLEN et al., 1998). Embora seja considerado o método com melhor desempenho na estimativa da $\mathrm{ET}_{0}$, apresenta restrições quanto à grande quantidade de informações necessárias para tal (PALARETTI et al., 2014a). Este método exige vários parâmetros de entrada como temperatura do ar, umidade relativa, radiação solar e velocidade do vento. Entretanto, há um número limitado de estações meteorológicas para monitoramento dessas variáveis do tempo (CUNHA et al., 2013) ou não dispõem de instrumentos necessários para a medição desses elementos, e quando dispõem desses instrumentos, mesmo assim, ocorrem falhas na coleta dos dados (SILVA et al., 2010).

Além das poucas estações meteorológicas na região Nordeste, e mais particularmente no estado do Ceará, é comum em algumas estações séries de dados de radiação solar sem continuidade, ou velocidade do vento, dentre outros, que justifica plenamente o uso de outros métodos que requerem menos dados meteorológicos (SILVA et al., 2014a). Uma alternativa seria o método de HARGREAVES \& SAMANI (1985) que tem apresentando bons resultados em regiões onde há escassez de dados, sendo uma boa alternativa para evitar um grande desperdício de água no momento de aplicar uma determinada lâmina de irrigação (SILVA et al., 2010).

Em uma abordagem apresentada por CARVALHO et al. (2011) de trabalhos mais recentes sobre desempenho de métodos de estimativa da $\mathrm{ET}_{\mathrm{o}}$, concluíram que, quando há disponibilidade de dados para o método de Penman-Monteith FAO56 (PM-FAO 56), este deve ser o recomendado como padrão, tendo em vista sua comprovada precisão, independentemente da escala temporal e espacial. Caso contrário, deve-se averiguar um método que esteja de acordo com os dados disponíveis e se é adequado às condições climáticas do local.

Vários estudos foram realizados a partir do método de HARGREVES \& SAMANI (1985) para a estimativa da $\mathrm{ET}_{0}$ em diferentes localidades. Nas condições 
climáticas de Mossoró, RN este método foi recomendado para a estimativa da $\mathrm{ET}_{\mathrm{o}}$ mensal (CAVALCANTE JUNIOR et al., 2010; CAVALCANTE JUNIOR et al., 2011) e diária (BEZERRA et al., 2014) quando se dispõem apenas de dados de temperatura do ar. No estado da Bahia, nas localidades de Eunápolis (TAGLIAFERRE et al., 2010); Anagé, Piatã e Ilhéus (TAGLIAFERRE et al., 2012) e Rio Real (CHAGAS et al., 2013), independente das condições climáticas estudadas (semiárida ou subúmida), o método de HARGREAVES \& SAMANI (1985) se destacou como sendo o pior método para a estimativa da $\mathrm{ET}_{\mathrm{o}}$. Já na localidade de Juazeiro, este o método foi recomendado para estimativa da $\mathrm{ET}_{\mathrm{o}}$ por apresentar desempenho próximo ao método padrão (PM-FAO 56) (OLIVEIRA et al., 2010).

\section{MATERIAL E MÉTODOS}

O trabalho foi realizado no estado do Ceará, que se encontra na região Nordeste do Brasil, entre os paralelos $2,5^{\circ}$ e $8^{\circ}$ de latitude Sul e os meridianos $37^{\circ}$ e $42^{\circ}$ de longitude Oeste. De acordo com a classificação climática de Köppen, a região em estudo apresenta três tipos de clima: BSw'h', Aw' e Cw', havendo uma predominância em aproximadamente $80 \%$ da área como BSw'h'(semiárido quente) (OLIVEIRA et al., 2013). Obteve-se dados diários de temperatura máxima e mínima do ar, umidade relativa, velocidade do vento e insolação, oriundos da rede de
No estado do Ceará em trabalhos anteriores, o método de HARGREAVES \& SAMANI (1985) foi recomendado para a estimativa da $\mathrm{ET}_{\mathrm{o}}$ diária na localidade de Sobral (GONÇALVES et al., 2009) e para escala mensal nas localidades de Crateús e Jaguaruana (ARAÚJO et al., 2010); já nas localidades de Guaramiranga (ARAÚJO et al., 2010), Fortaleza (ALMEIDA et al., 2010) e Campos Sales (FERNANDES et al., 2012a; SILVA et al., 2014a), este método não é recomendado.

Diante do exposto, objetivou-se com o presente trabalho avaliar a estimativa da evapotranspiração de referência mensal a partir do método de HARGREAVES \& SAMANI (1985) em comparação ao método padrão Penman-Monteith (FAO56), nas condições climáticas do estado do Ceará.

estações meteorológicas convencionais do estado, disponibilizados pelo Instituto Nacional de Meteorologia (INMET), (Tabela 1 e Figura 1). Com o auxílio de planilhas eletrônicas os dados foram organizados em escala de tempo mensal.

Para a estimativa da $\mathrm{ET}_{0}$ foi utilizado o método proposto por HARGREAVES \& SAMANI (1985) (Equação 1) e para avaliar o seu desempenho foi empregado o método de Penman-Monteith, parametrizado pela FAO no seu manual número 56 (ALLEN et al., 1998), (Equação 2).

$$
\mathrm{ET}_{\mathrm{o}} \mathrm{HS}=0,0023\left(\mathrm{~T}_{\text {máx }}-\mathrm{T}_{\text {mín }}\right)^{0,5}\left(\mathrm{~T}_{\text {méd }}+17,8\right) \cdot \mathrm{R}_{\mathrm{a}} \cdot 0,408
$$

em que: $\mathrm{ET}_{0} \mathrm{HS}$ é a evapotranspiração de referência pelo método de HARGREAVES \& SAMANI (1985), $\mathrm{mm} \mathrm{dia}^{-1} ; \mathrm{T}_{\text {máx }}$ é a temperatura máxima diária do ar, ${ }^{\circ} \mathrm{C} ; \mathrm{T}_{\text {mín }}$ é a temperatura mínima diária do ar, ${ }^{\circ} \mathrm{C} ; \mathrm{T}_{\text {méd }}$ é a temperatura média diária do ar, ${ }^{\circ} \mathrm{C} ; \mathrm{R}_{\mathrm{a}}$ é a radiação no topo da atmosfera, $\mathrm{MJ} \mathrm{\textrm {m } ^ { - 2 }}$ $\mathrm{dia}^{-1}$.

$$
\mathrm{ET}_{\mathrm{o}} \mathrm{PM}=\frac{0,408 \Delta\left(\mathrm{R}_{\mathrm{n}}-\mathrm{G}\right)+\gamma \frac{900}{\mathrm{~T}_{\mathrm{m}}+273} \mathrm{u}_{2}\left(\mathrm{e}_{\mathrm{s}}-\mathrm{e}_{\mathrm{a}}\right)}{\Delta+\gamma\left(1+0,34 \cdot \mathrm{u}_{2}\right)}
$$


em que: $\mathrm{ET}_{0} \mathrm{PM}$ é a evapotranspiração de referência pelo método de PenmanMonteith, $\mathrm{mm} \mathrm{dia}^{-1} ; \mathrm{R}_{\mathrm{n}}$ é a radiação líquida total, $\mathrm{MJ} \mathrm{m}^{-2} \mathrm{dia}^{-1} ; \mathrm{G}$ é densidade do fluxo de calor no solo, $\mathrm{MJ} \mathrm{m}^{-2} \mathrm{dia}^{-1} ; \mathrm{T}_{\text {méd }}$ é a temperatura média diária do ar, ${ }^{\circ} \mathrm{C} ; \mathrm{u}_{2}$ é a velocidade do vento média diária a $2 \mathrm{~m}$ de altura, $\mathrm{m} \mathrm{s}^{-1} ; \mathrm{e}_{\mathrm{s}}$ é a pressão de saturação de vapor, $\mathrm{kPa} ; \mathrm{e}_{\mathrm{a}}$ é a pressão parcial de vapor, $\mathrm{kPa} ; \mathrm{e}_{\mathrm{s}}-\mathrm{e}_{\mathrm{a}}$ é o déficit de saturação de vapor, $\mathrm{kPa} ; \Delta$ é a declividade da curva de pressão de vapor no ponto de $\mathrm{T}_{\text {méd }}, \mathrm{kPa}^{\circ} \mathrm{C}^{-1} ; \gamma$ é o coeficiente psicrométrico, $\mathrm{kPa}^{\circ} \mathrm{C}^{-1}$.

Tabela 1. Localização das estações meteorológicas monitoradas pelo INMET, no estado do Ceará

\begin{tabular}{|c|c|c|c|c|c|}
\hline Código & Estação & Latitude & Longitude & Altitude (m) & Período \\
\hline 82294 & Acaraú & $-2^{\circ} 53^{\prime} 08^{\prime \prime}$ & $-40^{\circ} 07^{\prime} 12^{\prime \prime}$ & 7,32 & $1976-2011$ \\
\hline 82392 & Sobral & $-3^{\circ} 41^{\prime} 10^{\prime \prime}$ & $-40^{\circ} 20^{\prime} 59^{\prime \prime}$ & 69,4 & $1961-2012$ \\
\hline 82397 & Fortaleza & $-3^{\circ} 43^{\prime} 02^{\prime \prime}$ & $-38^{\circ} 32^{\prime} 35^{\prime \prime}$ & 21,1 & $1963-2012$ \\
\hline 82487 & Guaramiranga & $-4^{\circ} 15^{\prime} 48^{\prime \prime}$ & $-38^{\circ} 55^{\prime} 59^{\prime \prime}$ & 900,0 & $1961-2012$ \\
\hline 82493 & Jaguaruana & $-4^{\circ} 52^{\prime} 02^{\prime \prime}$ & $-37^{\circ} 46^{\prime} 52^{\prime \prime}$ & 12,2 & 1969-2012 \\
\hline 82583 & Crateús & $-5^{\circ} 10^{\prime} 42^{\prime \prime}$ & $-40^{\circ} 40^{\prime} 39^{\prime \prime}$ & 274,7 & $1963-2012$ \\
\hline 82586 & Quixeramobim & $-5^{\circ} 11^{\prime} 57^{\prime \prime}$ & $-39^{\circ} 17^{\prime} 34^{\prime \prime}$ & 191,7 & 1961-2012 \\
\hline 82588 & Morada Nova & $-5^{\circ} 06^{\prime} 24^{\prime \prime}$ & $-38^{\circ} 22^{\prime} 21^{\prime \prime}$ & 52,1 & $1962-2012$ \\
\hline 82683 & Tauá & $-6^{\circ} 00^{\prime} 11^{\prime \prime}$ & $-40^{\circ} 17^{\prime} 34^{\prime \prime}$ & 402,6 & 1964-2012 \\
\hline 82686 & Iguatu & $-6^{\circ} 21^{\prime} 34^{\prime \prime}$ & $-39^{\circ} 17^{\prime} 55^{\prime \prime}$ & 217,2 & $1961-2012$ \\
\hline 82777 & Campos Sales & $-7^{\circ} 04^{\prime} 28^{\prime \prime}$ & $-40^{\circ} 22^{\prime} 34^{\prime \prime}$ & 566,3 & $1963-2012$ \\
\hline 82784 & Barbalha & $-7^{\circ} 18^{\prime} 40^{\prime \prime}$ & $-39^{\circ} 18^{\prime} 15^{\prime \prime}$ & 415,0 & 1979-2012 \\
\hline
\end{tabular}

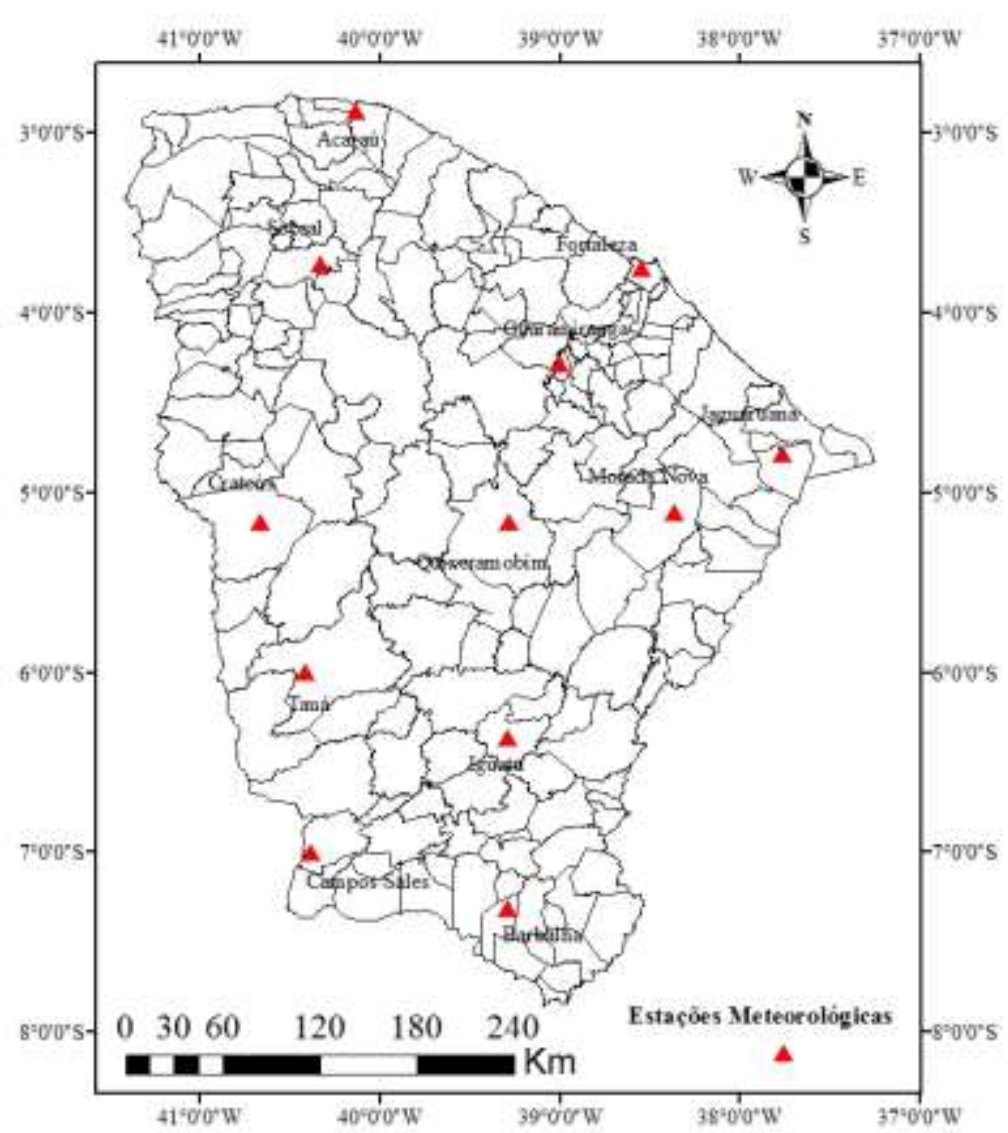

Figura 1. Distribuição espacial das estações meteorológicas no estado do Ceará.

Fonte: LEDO et al. (2013). 
A comparação dos valores de $\mathrm{ET}_{\mathrm{o}}$ obtidos pelo método padrão PenmanMonteith FAO 56 e pelo método de HARGREAVES \& SAMANI (1985) foi realizada usando o erro padrão de estimativa (EPE) (Equação 3) e a razão entre as médias ( $\zeta$ ), que representa subestimativa ou superestimativa (Equação 4). Utilizou-se também o índice "d" de concordância ou exatidão (WILLMOTT et al., 1985), onde seus valores variam de " 0 " (não existe concordância) a "1" (concordância perfeita) (Equação 5) e como parâmetro estatístico de comparação, utilizou-se $o$ índice de confiança ou desempenho "c", proposto por CAMARGO \& SENTELHAS (1997), reunindo os índices de precisão " $\mathrm{r}$ " $\mathrm{e}$ de exatidão “d”, sendo expresso por (c = r.d).

$$
\begin{aligned}
& \mathrm{EPE}=\left[\frac{\sum(\mathrm{Pi}-\mathrm{Oi})^{2}}{\mathrm{n}-1}\right]^{0,5} \\
& \zeta=\frac{\mathrm{P}}{\mathrm{O}} \cdot 100 \\
& \mathrm{~d}=1-\left[\frac{\sum_{\mathrm{i}=1}^{\mathrm{n}}(\mathrm{Pi}-\mathrm{Oi})^{2}}{\sum_{\mathrm{i}=1}^{\mathrm{n}}[(|\mathrm{Pi}-\mathrm{O}|)+(|\mathrm{Oi}-\mathrm{O}|)]^{2}}\right]
\end{aligned}
$$

em que: EPE é o erro padrão de estimativa, $\mathrm{mm} \mathrm{dia}^{-1}$; Pi é a $\mathrm{ET}_{\mathrm{o}}$ estimada pelo método de HARGREAVES \& SAMANI (1985), $\mathrm{mm} \mathrm{dia}^{-1}$; Oi é a $\mathrm{ET}_{\mathrm{o}}$ estimada pelo método de Penman-Monteith, $\mathrm{mm} \mathrm{dia}^{-1}$; $\mathrm{P}$ é a média da $\mathrm{ET}_{\mathrm{o}}$ estimada pelo método de HARGREAVES \& SAMANI (1985), mm dia $^{-1}$; O é a média da $\mathrm{ET}_{\mathrm{o}}$ estimada pelo método de Penman-Monteith, mm dia ${ }^{-1} ; \xi$ é

\section{RESULTADOS E DISCUSSÃO}

Buscou-se com o presente trabalho avaliar a relação entre os valores mensais de $\mathrm{ET}_{\mathrm{o}}$ estimados pelo método padrão Penman-Monteith FAO 56 (PM-FAO 56) e o método de HARGREAVES \& SAMANI (1985) (HS) que envolve menor quantidade de parâmetros de entrada, de modo a se obter de forma mais simples a $\mathrm{ET}_{\mathrm{o}}$ no estado do Ceará.

Nas localidades de Guaramiranga, Barbalha e Sobral ocorreram as maiores superestimativas da $\mathrm{ET}_{\mathrm{o}}$ estimada pelo método de HS em relação ao método a razão entre as médias, \%; n é o número de observações.

O índice de desempenho "c" é interpretado e distribuído nas seguintes classes: "Ótimo", c $>0,85$; "Muito Bom", $0,76 \leq \mathrm{c} \leq 0,85$; "Bom", $0,66 \leq \mathrm{c} \leq 0,75$; "Mediano", $0,61 \leq \mathrm{c} \leq 0,65$; "Sofrível", $0,51 \leq \mathrm{c} \leq 0,60$; "Mau", $0,41 \leq \mathrm{c} \leq 0,50$ e "Péssimo", c $\leq 0,40$ (CAMARGO \& SENTELHAS, 1997).

padrão PM-FAO 56, expressas pela razão entre as médias $(\xi)$, quais sejam da ordem de 17,$76 ; 12,16$ e $10,58 \%$, respectivamente (Tabela 2). Resultados similares foram reportados por PALARETTI et al. (2014b) em diferentes regiões citrícolas paulistas, em que o método de HS tendeu a superestimar a $\mathrm{ET}_{\mathrm{o}}$ padrão. Tendência de superestimativa de $\mathrm{ET}_{\mathrm{o}}$ padrão foi reportado por FERNANDES et al. (2012b) no estado de Goiás, no entanto, aqueles autores calibraram os coeficientes do método de HS de maneira local e regional. 
Em localidades do estado de Minas Gerais (ALENCAR et al., 2015) e em diferentes localidades na região do Sudeste brasileiro (CARVALHO et al., 2015), o método de HS apresentou maiores erros quando comparado com o método de PM-FAO56, com superestimativa da $\mathrm{ET}_{\mathrm{o}}$ diária.

Considerando a variabilidade climática entre as localidades em estudo e a divergência entre os resultados encontrados de $\mathrm{ET}_{\mathrm{o}}$ pelo método de HARGREAVES \& SAMANI (1985), justifica-se que, originalmente o método de foi desenvolvido para clima semiárido, e como se baseia apenas em dados de temperatura, espera-se que este método superestime a $\mathrm{ET}_{\mathrm{o}}$ em climas úmidos.

Tabela 2. Indicadores estatísticos da comparação da evapotranspiração de referência pelo método padrão de Penman-Monteith FAO-56 e pelo método de HARGREAVES \& SAMANI (1985), em diferentes localidades no estado do Ceará.

\begin{tabular}{cccccc}
\hline \multirow{2}{*}{ Localidades } & \multicolumn{5}{c}{ Indicadores estatísticos } \\
\cline { 2 - 6 } & $\boldsymbol{\xi}(\mathbf{\%})$ & EPE $\left(\mathbf{m m ~ d i a}^{-\mathbf{1}}\right)$ & $\mathbf{d}$ & $\mathbf{c}$ & Desempenho \\
\hline Acaraú & 102,29 & 0,54 & 0,77 & 0,59 & Sofrível \\
Barbalha & 112,16 & 0,71 & 0,79 & 0,64 & Mediano \\
Campos Sales & 99,37 & 0,88 & 0,74 & 0,49 & Mau \\
Crateús & 103,45 & 0,66 & 0,85 & 0,73 & Bom \\
Fortaleza & 89,19 & 0,77 & 0,59 & 0,32 & Péssimo \\
Guaramiranga & 117,76 & 0,64 & 0,76 & 0,65 & Mediano \\
Iguatu & 96,91 & 0,63 & 0,88 & 0,74 & Bom \\
Jaguaruana & 101,54 & 0,58 & 0,86 & 0,73 & Bom \\
Morada Nova & 104,31 & 0,66 & 0,86 & 0,67 & Bom \\
Quixeramobim & 92,09 & 0,93 & 0,80 & 0,69 & Bom \\
Sobral & 110,58 & 0,85 & 0,81 & 0,67 & Bom \\
Tauá & 107,67 & 0,71 & 0,79 & 0,60 & Sofrível \\
\hline
\end{tabular}

$\mathrm{Na}$ localidade de Campos Sales ocorreu leve subestimativa da $\mathrm{ET}_{\mathrm{o}}$, de apenas $0,63 \%$, ou seja, a média da $\mathrm{ET}_{\mathrm{o}}$ estimada por HS e PM-FAO 56 foi praticamente da mesma ordem. Subestimativa da $\mathrm{ET}_{\mathrm{o}}$ também foi verificada em Fortaleza, Iguatu e Quixeramobim, respectivamente da ordem de 10,81, 3,09 e 7,91\%. Em Uberlândia, MG, LACERDA \& TURCO (2015) reportaram subestimativa da $\mathrm{ET}_{\mathrm{o}}$ diária por HS da ordem de $11 \%$ em relação ao método padrão de PM-FAO 56.

Outro índice estatístico utilizado na comparação das estimativas de $\mathrm{ET}_{\mathrm{o}}$ foi o erro padrão de estimativa (EPE), que representa uma medida da magnitude média dos erros estimados, com valor sempre positivo e quanto mais próximo de zero, maior a qualidade dos valores medidos ou estimados. O menor EPE foi na localidade de Acaraú, da ordem de 0,54 $\mathrm{mm} \mathrm{dia}^{-1}$ e o maior EPE de $0,93 \mathrm{~mm} \mathrm{dia}^{-1}$ em Quixeramobim.

Quanto ao índice de exatidão ou concordância "d" e o índice de desempenho "c", os maiores índices foram encontrados nas localidades de Iguatu ("d" $=0,88$ e "c" = 0,74; "Bom"), Jaguaruana ("d" = 0,86 e "c" = 0,73; "Bom") e Crateús ("d" = 0,85 e "c" = 0,73; "Bom"). Desempenho classificado como "Bom" também foi verificado nas localidades de Morada Nova, Quixeramobim e Sobral, porém com valores de "c" inferiores aos citados acima, o que pode ser justificado pela amplitude da classe conforme a tabela proposta por CAMARGO \& SENTELHAS (1997). Em trabalho realizado anteriormente em Sobral por GONÇALVES et al. (2009), o desempenho do método de HS foi melhor ("c" = 0,76 ; "Muito Bom") ao apresentado no presente estudo, o que pode estar relacionado com a 
série de dados utilizada, pois no trabalho de GONÇALVES et al. (2009) a estimativa da $\mathrm{ET}_{\mathrm{o}}$ foi apenas com um ano de dados diários. Ainda de acordo com os autores, a boa confiabilidade deste método que utiliza apenas a temperatura do ar pode ser justificada pela variação de temperatura na região em estudo. No estado de Pernambuco, desempenho "Muito Bom" na estimativa da $\mathrm{ET}_{\mathrm{o}}$ diária por $\mathrm{HS}$ foi reportado por SILVA et al. (2012) nas condições climáticas de Recife e na microrregião de Garanhuns dentre os métodos baseados na temperatura do ar, o de HS apresentou o melhor desempenho (BORGES JÚNIOR et al., 2012).

Assim como constatado no presente trabalho, nas condições climáticas de Juazeiro, BA ("d" = 0,85 e "c" = 0,67 ) (OLIVEIRA et al., 2010), em Mossoró, RN (CAVALCANTE JUNIOR et al., 2010; CAVALCANTE JUNIOR et al., 2011; BEZERRA et al., 2014), em Jales, $\mathrm{SP}$ ("d" = $0,76 \mathrm{e}$ "c" = 0,69 ) (CONCEIÇÃO et al., 2013) e em Nova Venécia, ES no período seco ("d" = 0,96 e "c" = 0,69) (SOUZA et al., 2014), o método de HS apresentou desempenho "Bom" na estimativa da $\mathrm{ET}_{\mathrm{o}}$ em relação ao método padrão.

Desempenho "Mediano" foi encontrado nas localidades de Barbalha ("d" $=0,79 \mathrm{e}$ "c" $=0,64)$ e Guaramiranga ("d" = 0,76 e "c" $=0,65)$. Resultados similares foram encontrados por TAGLIAFERRE et al. (2012), em que nas condições climáticas de Piatã, BA a $\mathrm{ET}_{\mathrm{o}}$ estimada por HS nas escalas diária, de três, cinco e sete dias superestimou a $\mathrm{ET}_{\mathrm{o}}$ padrão, com desempenho "Mediano" em todas as escalas.

Os piores índices foram encontrados em Acaraú ("d" = 0,77 e "c" = 0,59; "Sofrível"), Tauá ("d" = 0,79 e "c" = 0,60; "Sofrível"), Campos Sales ("d" = 0,74 e "c" = 0,49; "Mau") e Fortaleza ("d" = 0,59 e "c" = 0,32; "Péssimo"). Desempenho
"Sofrível" na estimativa da $\mathrm{ET}_{\mathrm{o}}$ pelo método de HS foi encontrado em outras regiões brasileiras, em Ilhéus (TAGLIAFERRE et al., 2012) e Rio Real, BA no período seco (CHAGAS et al., 2013) e em Aquidauana (RIGONI et al., 2013) e Dourados, MS (NOIA et al., 2014). Na localidade de Fortaleza, os resultados encontrados no presente estudo corroboram com trabalho anterior realizado por ALMEIDA et al. (2010), sendo que o uso do método de HS subestimou a $\mathrm{ET}_{\mathrm{o}}$ padrão mensal, com desempenho "Péssimo". Desempenho "Péssimo" foi confirmado por SILVA et al. (2014b) no Arquipélago de Fernando de Noronha, PE, em que o método de HS superestimou a $\mathrm{ET}_{\mathrm{o}}$ padrão nas escalas diária, cinco e dez dias, respectivamente. Nas condições climáticas de Campos dos Goytacazes, RJ, segundo FERNANDES et al. (2012c) o método de HS mostrou-se limitado para a estimativa da $\mathrm{ET}_{\mathrm{o}}$ nessa localidade.

Tais resultados nessas localidades, possivelmente estar associado ao número de variáveis utilizadas pelo método, uma vez, que o mesmo considera apenas a temperatura do ar e a radiação no topo da atmosfera na sua estimativa.

Considerando as variações significativas no desempenho do método de HS dentro do estado do Ceará, visando melhor desempenho desse método, em trabalhos futuros o ideal é ajustar os coeficientes empíricos da equação para a região de estudo. Logo, por meio da utilização da equação de HS ajustada, há a possibilidade de se fazer um manejo da irrigação utilizando-se somente as variáveis meteorológicas de temperaturas máximas e mínimas, as quais podem ser obtidas através de sensores economicamente acessíveis, com isso pode-se otimizar os recursos hídricos no momento de aplicar uma determinada lâmina de irrigação. 


\section{CONCLUSÕES}

Baseado nos resultados encontrados no presente estudo, quando se dispõem apenas de dados de temperatura do ar recomenda-se o método de HARGREAVES \& SAMANI (1985) para a estimativa da evapotranspiração de referência em substituição ao método padrão Penman-Monteith FAO-56, nas localidades de Crateús, Iguatu, Jaguaruana,

\section{REFERÊNCIAS}

ALLEN, R. G.; PEREIRA, L. S.; RAES, D.; SMITH, M. Crop evapotranspiration - Guidelines for computing crop water requirements. Roma: FAO, 1998. 300p. (FAO Irrigation and Drainage Paper, 56).

ALENCAR, L. P.; SEDIYAMA, G. C.; MANTOVANI, E. C. Estimativa da evapotranspiração de referência (ETo padrão FAO), para Minas Gerais, na ausência de alguns dados climáticos. Engenharia Agrícola, v.35(1): 39-50, 2015.

ALMEIDA, B. M.; ARAÚJO, E. M.; CAVALCANTE JUNIOR, E. G.; OLIVEIRA, J. B.; ARAÚJO, E. M.; NOGUEIRA, B. R. C. Comparação de métodos de estimativa da ETo na escala mensal em Fortaleza-CE. Revista Brasileira de Agricultura Irrigada, v.4(2): 93-98, 2010.

ARAÚJO, E. M.; OLIVEIRA， J. B.; ARAÚJO, E. M.; LÊDO, E. R. F.; SILVA, M. G.; Desempenho de métodos de estimativa de ETo correlacionados com a equação padrão Penman Monteith FAO56, em cidades do estado do Ceará. Revista Acta Tecnológica, v.5(2): 84-101, 2010.

BEZERRA, J. M.; MOURA, G. B. A.; SILVA, E. F. F.; LOPES, P. M. O.; SILVA, B. B. Estimativa da evapotranspiração de referência diária para Mossoró (RN, Brasil). Revista Caatinga, v.27(3): 211-220, 2014.

BORGES JÚNIOR, J. C. F.; ANJOS, R. J.; SILVA, T. J. A.; LIMA, J. R. S.; ANDRADE, C. L. T. Métodos de
Morada Nova, Quixeramobim e Sobral no estado do Ceará.

Nas localidades situadas em regiões litorâneas (Acaraú e Fortaleza) e serranas (Guaramiranga) o método de HARGREAVES \& SAMANI (1985) não é recomendado para a estimativa da evapotranspiração de referência.

estimativa da evapotranspiração de referência diária para a microrregião de Garanhuns, PE. Revista Brasileira de Engenharia Agrícola e Ambiental, v.16(4): 380-390, 2012.

CAMARGO, A. P.; SENTELHAS, P. C. Avaliação do desempenho de diferentes métodos de estimativa da evapotranspiração potencial no Estado de São Paulo. Revista Brasileira de Agrometeorologia, v.5(1): 89-97, 1997.

CARVALHO, L. G.; RIOS, G. F. A.; MIRANDA, W. L.; CASTRO NETO, P. C. Evapotranspiração de referência: uma abordagem atual de diferentes métodos de estimativa. Pesquisa Agropecuária Tropical, v.41(3): 456-465, 2011.

CARVALHO, D. F.; ROCHA, H. S.; BONOMO, R.; SOUZA, A. P. Estimativa da evapotranspiração de referência a partir de dados meteorológicos limitados. Pesquisa Agropecuária Brasileira, v.50(1): 1-11, 2015.

CAVALCANTE JUNIOR, E. G.; ALMEIDA, B. M.; OLIVEIRA, A. D.; ESPÍNOLA SOBRINHO, J.; ARAÚJO, E. M.; VIEIRA, R. Y. M. Estimativa da evapotranspiração de referência para a cidade de Mossoró-RN. Revista Brasileira de Agricultura Irrigada, v.4(2): 87-92, 2010.

CAVALCANTE JUNIOR, E. G.; OLIVEIRA, A. D.; ALMEIDA, B. M.; ESPINOLA SOBRINHO, J. Métodos de estimativa da evapotranspiração de referência para as condições do semiárido 
nordestino. Semina: Ciências Agrárias, v.32(1): 1699-1708, 2011.

CHAGAS, R. M.; FACCIOLI, G. G.; AGUIAR NETTO, A. O.; SOUSA, I. F.; VASCO, A. N.; SILVA, M. G. Comparação entre métodos de estimativa da evapotranspiração de referência (ETo) no município de Rio Real-BA. Irriga, v.18(1): 351-363, 2013.

CONCEIÇÃO, M. A. F. Ajuste do modelo de Hargreaves para estimativa da evapotranspiração de referência no noroeste paulista. Revista Brasileira de Agricultura Irrigada, v.7(5): 306-316, 2013.

CUNHA, F. F.; MAGALHÃES, F. F.; CASTRO, M. A. Métodos para estimativa da evapotranspiração de referência para Chapadão do Sul - MS. Engenharia na Agricultura, v.21(2): 159-172, 2013.

ESTEVES, B. S.; MENDONÇA, J. C.; SOUSA, E. F.; BERNARDO, S. Avaliação do Kt para estimativa da evapotranspiração de referência (ETo) em Campos dos Goytacazes, RJ. Revista Brasileira de Engenharia Agrícola e Ambiental, v.14(3): 274-278, 2010.

FERNANDES, D. S.; HEINEMANN, A. B.; PAZ, R. L. F.; AMORIM, A. O. Calibração regional e local da equação de Hargreaves para estimativa da evapotranspiração de referência. Revista Ciência Agronômica, v.43(2): 246-255, 2012b.

FERNANDES, J. L.; OLIVEIRA, J. B.; SOUZA, A. L. M.; SILVA, G. S.; IZIDIO, N. S. C. Avaliação de modelos de estimativa da evapotranspiração de referência em Campos Sales-CE. Conexões: Ciência e Tecnologia, v. 6(3): 58-67, 2012a.

FERNANDES, L. C.; PAIVA, C. M.; ROTUNNO FILHO, O. C. Evaluation of six empirical evapotranspiration equations - case study: Campos dos Goytacazes/RJ.

Revista Brasileira de Meteorologia, v.27(3): 272-280, 2012c.
GONÇALVES, F. M.; FEITOSA, H. O.; CARVALHO, C. M.; GOMES FILHO, R. R.; VALNIR JÚNIOR, M. Comparação de métodos da estimativa da evapotranspiração de referência para o município de Sobral-CE. Revista Brasileira de Agricultura Irrigada, v.3(2): 71-77, 2009.

LACERDA, Z. C.; TURCO, J. E. P. Estimation methods of reference evapotranspiration (ETo) for Uberlândia MG. Engenharia Agrícola, v.35(1): 2738, 2015.

LEDO, E. R. F.; SILVA, M. G.; SANTOS, N. T.; SILVA FILHO, J. A.; BATISTA, L. S. Espacialização do balanço hídrico no Ceará. Parte I: Cenário atual. In: Anais... XLII Congresso Brasileiro de Engenharia Agrícola - CONBEA, 2013, Fortaleza.

MENDONÇA, E. A.; DANTAS, R. T. Estimativa da evapotranspiração de referência no município de Capim, PB. Revista Brasileira de Engenharia Agrícola e Ambiental, v.14(2): 196-202, 2010 .

MINUZZI, R. B.; RIBEIRO, A. J.; SILVA, D. O.; KUNESKI, A. C. Estimativa da evapotranspiração de referência diária por Penman-Monteith FAO com dados de temperatura do ar para Santa Catarina. Irriga, v.19(3): 548-558, 2014.

NOIA, C. P. Z.; PEREIRA, S. B.; ROSA, D. R. Q.; ALMEIDA, R. A. Evapotranspiração de referência estimada pelos métodos Penman-Monteith-FAO (56) e Hargreaves \& Samani para o município de Dourados, MS. Revista Agrarian, v.7(24): 300-308, 2014.

OLIVEIRA, G. M.; LEITÃO, M. M. V. B. R.; BISPO, R. C.; SANTOS, I. M. S.; ALMEIDA, A. C. Comparação entre métodos de estimativa da evapotranspiração de referência na região norte da Bahia. Revista Brasileira de Agricultura Irrigada, v.4(2): 104-109, 2010.

OLIVEIRA, J. B.; ARRAES, F. D. D.; VIANA, P. C. Methodology for the spatialisation of a reference 
evapotranspiration from SRTM data. Revista Ciência Agronômica, v.44(3): 445-454, 2013.

PALARETTI, L. F.; MANTOVANI, E. C.; SEDIYAMA, G. C. Análise da sensibilidade dos componentes da equação de Hargreaves-Samani para a região de Bebedouro-SP. Revista Brasileira de Meteorologia, v.29(2): 299-306, 2014a.

PALARETTI, L. F.; MANTOVANI, E. C.; SEDIYAMA, G. C. Comparação entre métodos de estimativa da evapotranspiração de referência (ETo) em regiões citrícolas paulistas. Engenharia Agrícola, v.34(1): 38-47, 2014b.

RIGONI, E. R.; OLIVEIRA, G. Q.; BISCARO, G. A.; QUEIRÓZ, M. V. B. M.; LOPES, A. S. Desempenho sazonal da evapotranspiração de referência em Aquidauana, MS. Engenharia na Agricultura, v.21(6): 547-562, 2013.

SILVA, A. O.; SILVA, E. F. F.; MOURA, G. B. A.; LOPES, P. M. O. Avaliação do desempenho de métodos de estimativa de evapotranspiração potencial para a região Norte de Recife-PE. Engenharia na Agricultura, v.20(2): 163-174, 2012.

SILVA, J. R. L.; MONTENEGRO, A. A. A.; SANTOS, T. E. M.; SANTOS, E. S. Desempenho de diferentes métodos de estimativa da evapotranspiração de referência para Fernando de Noronha. Irriga, v.19(3): 390-404, 2014b.

SILVA, M. G.; LÊDO, E. R. F.; SANTOS, N. T.; CARMO, F. F.; SOUZA, E. A.; BATISTA, L. S. Métodos de estimativa da evapotranspiração de referência mensal para a Microrregião da Chapada do Araripe, Ceará. In: Anais... II Inovagri International Meeting, 2014a, Fortaleza. p.331-340.

SILVA, M. G.; OLIVEIRA, J. B.; LÊDO, E. R. F.; ARAÚJO, E. M.; ARAÚJO, E. M. Estimativa da $\mathrm{ET}_{\mathrm{o}}$ pelos métodos Penman-Monteith FAO 56 e HargreavesSamani a partir de dados de Tx e Tn para Sobral e Tauá no Ceará. Revista Acta Tecnológica, v.5(2): 52-68, 2010.
SILVA, V. P. R.; GARCÊZ, S. L. A.; SILVA, B. B.; ALBUQUERQUE, M. F.; ALMEIDA, R. S. R. Métodos de estimativa da evapotranspiração da cultura da cana-de-açúcar em condições de sequeiro. Revista Brasileira de Engenharia Agrícola e Ambiental, v.19(5): 411-417, 2015.

SOUZA, J. M.; PEREIRA, L. R.; RAFAEL, A. M.; SILVA, L. D.; REIS, E. F.; BONOMO, R. Comparison of methods for estimating reference evapotranspiration in two locations of Espirito Santo. Revista Brasileira de Agricultura Irrigada, v.8(2): 114-126, 2014.

TAGLIAFERRE， C.; SILVA， J. P.; PAULA, A.; GUIMARAES, D. U. G.; BARROSO, N. I. S. Estimativa da evapotranspiração de referência para três localidades do estado da Bahia. Revista Caatinga, v.25(2): 136-143, 2012.

TAGLIAFERRE, C.; SILVA, R. A. J.; ROCHA, F. A.; SANTOS, L. C.; SILVA, C. S. Estudo comparativo de diferentes metodologias para determinação da evapotranspiração de referência em Eunápolis-BA. Revista Caatinga, v.23(1): 103-111, 2010.

TRAJKOVIĆ, S.; STOJNIĆ, V.; GOCIĆ, M. Minimum weather data requirements for estimating reference evapotranspiration. Architecture and Civil Engineering, v.9(2): 335-345, 2011. 